\title{
A Systematic Review and Meta-Analysis of Mindfulness-Based (Baduanjin) Exercise for the Rehabilitation of Stroke Patients
}

Xiyan Xie ( $\sim 605999337 @ q q . c o m)$

Guizhou University of Finance and Economics

Research Article

Keywords: Baduanjin, stroke, rehabilitation, randomized controlled trial

Posted Date: December 14th, 2021

DOI: https://doi.org/10.21203/rs.3.rs-1121363/v1

License: (1) This work is licensed under a Creative Commons Attribution 4.0 International License. Read Full License 


\begin{abstract}
Background: Baduanjin is a fitness routine that has been developed in accordance with the appeal of the General Administration of Sport of China and promoted both in China and abroad. This paper aims to systematically evaluate the effect of Baduanjin on the rehabilitation of stroke patients.
\end{abstract}

Methods: We searched the Web of Science, Cochrane Library, Wanfang Database, Pubmed, Embase, CNKI, and VIP Chinese Journal Database for randomized controlled trials (RCTs) evaluating the rehabilitation effect of Baduanjin exercise on stroke patients. All manuscripts published before October 30,2021 were considered.

Results: A total of 1332 stroke patients were included in the 14 selected RCTs. Results of the meta-analysis showed that Baduanjin effectively improved the lower extremity balance function [MD $=9.74,95 \% C l(5.32,14.15), P<0.0001]$, sensory-motor function $[\mathrm{MD}=5.37,95 \% \mathrm{Cl}(1.59,9.16), P=0.005]$, neurological function [MD $=-2.41,95 \% C l(-3.07,-1.75), P<0.00001]$, the ability to perform daily activities [MD $=14.00,95 \% C l(6.17,21.83), P=0.0005]$, sleep quality [MD $=$ $-3.08,95 \% C l(-3.90,-2.25), P<0.00001]$, and quality of life [MD $=31.71,95 \% C l(13.68,49.73), P=0.06]$. Compared to conventional treatment, the rehabilitation effect was greater with Baduanjin combined with conventional treatment.

Conclusions: Baduanjin is an effective method to treat stroke patients and improve their functional performance. However, due to the small sample size and low data quality, further multicenter RCTs with large sample sizes are required to verify the results.

\title{
Background
}

Stroke, also known as apoplexia, cerebrovascular accident, and cerebral apoplexy is a brain disease caused by motor neuron injury, improving the spinal cord reflex activity, abnormal vascular function complications, leading to cell death, which neural disorder is the main reason for blockage of the cerebral blood flow, cerebral vascular obstruction, ischemic stroke, cerebrovascular rupture, and hemorrhagic stroke. Stroke is manifested by loss of proprioception, changes in coordination and balance patterns, resulting in balance and sensorimotor disorders and flexor spasm and hemiplegia in severe cases, and $30 \%$ of the stroke patients suffer from depression, leading to a reduction in their quality of life [1].

Two-thirds of stroke patients in the United States require rehabilitation [2]. The number of stroke cases in China is over 7 million, and about $70 \%-80 \%$ are ischemic stroke patients. Sequelae in surviving stroke patients lead to loss of function and $15 \%-30 \%$ of the stroke patients are permanently incapacitated every year, requiring treatment to improve the symptoms of stroke [3]. In China, the annual economic burden of treatment and care of stroke patients is about 10 billion Yuan [3]. Although immediate treatment reduces the recurrence and disability, newer methods to prevent and treat stroke are needed. Currently, oral and injectable muscle relaxants, gloves, orthoses, physical therapy, and surgical intervention can alleviate the symptoms, but these methods are expensive, require long-term intervention, and only emphasize the physical function, failing to treat symptoms such as depression, mood disorders, and anxiety. Therefore, newer more econoomic methods that alleviate depression and promote speedy rehabilitation of stroke patients are required. Regular physical activity is associated with a reduced risk of stroke [4]. Baduanjin part of China's intangible cultural heritage [5], is a traditional Chinese national fitness method that combines traditional Chinese medicine and Yin-Yang and Five elements. The physical and cognitive requirements of Baduanjin are low. Baduanjin strengthens the body, cultivates the minds, improves overall health, helps realize the integration of body and mind, facilitates the activity of the joints and muscles of the whole body, and enhances respiratory function. Targeted randomized controlled trials (RCTs) have verified the efficacy and feasibility of Baduanjin. The current study systematically reviewed the studies on the efficacy of Baduanjin in the rehabilitation of stroke patients, to provide concrete evidence for the application of Baduanjin in medical treatment of stroke patients, identify the limitations of current research, and provide feasible suggestions for further clinical research on the rehabilitation of stroke patients.

\section{Methods \\ Search strategy}

The following databases were used for literature retrieval: Web of Science, Cochrane Library, WanFang Data, Pubmed, Embase, China National Knowledge Internet (CNKI), and China Science and Technology Journal Database (VIP). The keywords used for obtaining the studies were: "Baduanjin", "Fitness Qigong", "Stroke”, "Cerebrovascular Accident”, "Cerebrovascular”, "Apoplexy”, “Cerebrovascular Apoplexy”, "Vascular Accident”, "Brain Vascular Accident”, “Cerebrovascular Stroke”, “Cerebral Stroke”, “Acute Stroke”, “Acute Cerebrovascular Accident”, “Function evaluation”, "sensory motor function”, "dysfunction”, "treatment", "rehabilitation", and "balance". Relevant search terms were combined using Boolean operators (OR/AND) according to the three search levels of cross-reference retrieval, identifying relevant experimental information in accordance with the Preferred Reporting Items for Systematic Reviews and Meta Analyses (PRISMA guidelines).

\section{Inclusion Criteria and Study Selection}

The criteria for selecting the stroke patients were cerebral infarction or cerebral hemorrhage confirmed using MRI or CT. There were no limitations on the course of the disease and pathological changes, patients' age, and frequency and course of treatment. The experimental (Baduanjin) group was compared with the control group. In the different studies, Baduanjin intervention was accompanied by balance training, medication, and walking intervention, and balance function, motor function, quality of life, anxiety, and depression were evaluated. There were no restrictions on routine rehabilitation methods for the control group. Primary outcomes included Berg Balance Scale (BBS), Fugl-Meyer Assessment Lower extremity (FMA-LE), Barthel index, Pittsburgh Sleep Quality Index, World Health Organization Quality of Life (WHOQOL), 36-item Short Form Survey (SF-36), Stroke-Specific Quality of Life Scale (SSQOL), and National Institutes of Health Stroke Scale (NIHSS). Exclusion criteria for studies were irrelevant, study objective, reviews, non RCTs, experience summaries, 
case reports, unavailability of full-text, conference papers, dissertations, data cannot be applied, studies with inconsistent indicators, observational studies, unpublished papers, duplicate publications, and animal studies.

\section{Literature Screening}

All related studies were retrieved using EndNote X9 and duplicate and similar studies by the same author or groups were not considered. Two researchers screened the studies according to inclusion and exclusion criteria by going through the title and abstract, and double-checked them. Differences between the researchers regarding the inclusion of a paper were resolved by a third researcher by going through the full text of the paper.

Figure 1 Flow diagram of study design

\section{Data extraction}

Two researchers extracted information from the papers, including study details (name of the principal author, publication date, and type of experimental design), participant characteristics (sample size and participation), sampling methods and procedures, general information of participants (age range and course of disease), intervention plan (practice time, place, and intensity), inclusion and exclusion criteria of subjects, and results. If standard deviation was not mentioned, $t$ value and confidence intervals were used to calculate the standard deviation. Basic information on the included papers was extracted, including sample size, age range, duration of disease, ischemia/ bleeding conditions, interventions, main observational indicators, and results.

\section{Quality evaluation of the studies}

Cochrane bias risk assessment tool was used to evaluate the quality and risk of bias of included studies [6]. The risk of bias was evaluated using 6 aspects of selection bias, including random sequence generation (selection bias), allocation concealment (selection bias), blinding of participants and personnel (performance bias), blinding of outcome assessment (detection bias), incomplete outcome data (attrition bias), selective reporting (reporting bias). The papers were categorized based on the degree of risk of bias into "low risk", "high risk", and "unclear risk". Two researchers evaluated the included papers according to these criteria, and in case of a disagreement, a third researcher participated in the evaluation procedure and negotiated a settlement.

\section{Statistical analysis}

A meta-analysis of RCTs with small measurement heterogeneity was performed using RevMan 5.3 software. Descriptive analysis of non-conflable data, heterogeneity test of included literature, heterogeneity was considered when $P<0.1$ or $P>50 \%$, fixed-effect models were used to evaluate sources that could be explained by clinical heterogeneity (low effect $=0.2$, medium effect $=0.5$, high effect $=0.8$ ), instead, use the random-effect model, $P<0.05$ for a significant difference, a $95 \%$ confidence interval was chosen for representation, heterogeneity was determined using Chi-square test and the variability of multiple study merger statistics by $Z$ test.

\section{Results}

Literature Search

A total of 166 articles were obtained from the literature search, of which, 14 publications

were finally considered for analysis. All manuscripts published before October 30, 2021 were considered. The screening procedure is shown in Figure 1.

\section{Study Characteristics}

Table 1 shows the characteristics of the selected 14 RCTs. All studies included trained professional Baduanjin participants. The intervention included combinations of Baduanjin training, balance training, Bobath therapy, music therapy, medication, and general rehabilitation training. The control group received Baduanjin training, Bobath therapy, music therapy, medication, and general rehabilitation training. Of the 1332 stroke patients, 565 did not report stroke type. Study sample sizes ranged from 40 to 221 patients. Intervention duration varied from 20 to 60 minutes per day, and it ranged from 3 weeks to 3 months. Seven studies used BBS, six used FMA-LE, four used Barthel index grading, two used PSQI, and WHOQOL, SF-36, SSQOL, and NIHSS were used by one study each. 
Table 1

Summary of the included studies

\begin{tabular}{|c|c|c|c|c|c|c|c|c|c|c|c|}
\hline \multirow{2}{*}{$\begin{array}{l}\text { Author } \\
\text { [Reference] }\end{array}$} & \multicolumn{2}{|c|}{ Sample } & \multicolumn{2}{|c|}{ Age range (year) } & \multicolumn{2}{|c|}{$\begin{array}{l}\text { course of disease } \\
\text { (month) }\end{array}$} & \multicolumn{2}{|c|}{$\begin{array}{l}\text { Ischemic/ } \\
\text { bleeding }\end{array}$} & \multicolumn{2}{|l|}{ Intervention } & \multirow[t]{2}{*}{ indicators } \\
\hline & EG & CG & EG & CG & EG & CG & EG & CG & EG & CG & \\
\hline $\begin{array}{l}\text { Cai et al. } \\
\text { [19] RCT }\end{array}$ & 30 & 30 & $60.27 \pm 10.48$ & $61.27 \pm 7.42$ & $7.20 \pm 2.38$ & $7.11 \pm 2.06$ & $21 / 9$ & $24 / 6$ & $\begin{array}{l}(4-5) \times 30 \\
\text { min/week } \\
\text { for } 3 \text { months } \\
+ \text { education } \\
\text { class }\end{array}$ & $\begin{array}{l}\text { education } \\
\text { class }\end{array}$ & WHOQOL \\
\hline $\begin{array}{l}\text { Cai et al. } \\
\text { [16] RCT }\end{array}$ & 30 & 30 & $60.27 \pm 10.48$ & $61.27 \pm 7.42$ & $7.20 \pm 2.38$ & $7.11 \pm 2.06$ & $21 / 9$ & $24 / 6$ & $\begin{array}{l}(4-5) \times 30 \\
\text { min/week } \\
\text { for } 3 \text { months } \\
+ \text { education } \\
\text { class }\end{array}$ & $\begin{array}{l}\text { education } \\
\text { class }\end{array}$ & $\mathrm{BI}$ \\
\hline $\begin{array}{l}\text { Bai et al. } \\
\text { [7] RCT }\end{array}$ & 30 & 30 & $53.7 \pm 4.5$ & $51.3 \pm 7.5$ & $1.21 \pm 0.16$ & $1.15 \pm 0.12$ & $18 / 12$ & $19 / 11$ & $\begin{array}{l}7 \times 40 \\
\text { min/week } \\
\text { for } 6 \text { weeks } \\
+ \text { balance } \\
\text { training }\end{array}$ & $\begin{array}{l}\text { balance } \\
\text { training }\end{array}$ & BBS \\
\hline $\begin{array}{l}\text { Guo et al. } \\
\text { [15] RCT }\end{array}$ & 115 & 106 & $57.5 \pm 24.5$ & $57.5 \pm 24.5$ & $3.5 \pm 2.5$ & $3.5 \pm 2.5$ & unclear & unclear & $\begin{array}{l}7 \times 40 \\
\text { min/week } \\
\text { for } 6 \text { weeks } \\
+ \text { Bobath } \\
\text { technologies } \\
\text { + routine } \\
\text { medication }\end{array}$ & $\begin{array}{l}\text { Bobath } \\
\text { technologies } \\
\text { + routine } \\
\text { medication }\end{array}$ & NIHSS \\
\hline $\begin{array}{l}\text { Zhang et } \\
\text { al. [12] } \\
\text { RCT }\end{array}$ & 115 & 106 & $57.5 \pm 24.5$ & $57.5 \pm 24.5$ & $3.5 \pm 2.5$ & $3.5 \pm 2.5$ & unclear & unclear & $\begin{array}{l}7 \times 40 \\
\text { min/week } \\
\text { for } 6 \text { weeks } \\
\text { + Bobath } \\
\text { technologies } \\
\text { + routine } \\
\text { medication }\end{array}$ & $\begin{array}{l}\text { Bobath } \\
\text { technologies } \\
\text { + routine } \\
\text { medication }\end{array}$ & BBS \\
\hline $\begin{array}{l}\text { Zhang et } \\
\text { al. [13] } \\
\text { RCT }\end{array}$ & 31 & 31 & $55.07 \pm 4.81$ & $56.72 \pm 3.57$ & $6.22 \pm 2.45$ & $7.01 \pm 1.89$ & $21 / 10$ & $19 / 12$ & $\begin{array}{l}5 \times 40 \\
\mathrm{~min} / \text { week } \\
\text { for } 8 \text { weeks } \\
+ \text { balance } \\
\text { training }+ \\
\text { rehabilitation }\end{array}$ & $\begin{array}{l}\text { balance } \\
\text { training + } \\
\text { rehabilitation }\end{array}$ & $\begin{array}{l}\text { BBS, } \\
\text { FMA-LE }\end{array}$ \\
\hline $\begin{array}{l}\text { Chen et al. } \\
\text { [20] RCT }\end{array}$ & 36 & 36 & $52.21 \pm 5.03$ & $51.83 \pm 4.87$ & $5.83 \pm 2.09$ & $6.35 \pm 1.69$ & $20 / 16$ & $22 / 14$ & $\begin{array}{l}7 \times 30 \\
\mathrm{~min} / \text { week } \\
\text { for } 40 \text { days }+ \\
\text { music } \\
\text { therapy }+ \\
\text { rehabilitation }\end{array}$ & $\begin{array}{l}7 \times 30 \\
\text { min/week } \\
\text { for } 40 \text { days + } \\
\text { rehabilitation }\end{array}$ & SF-36 \\
\hline $\begin{array}{l}\text { Tian [9] } \\
\text { RCT }\end{array}$ & 30 & 30 & $54.3 \pm 4.7$ & $53.0 \pm 4.3$ & unclear & unclear & $30 / 0$ & $30 / 0$ & $\begin{array}{l}2 \times(60-80) \\
\mathrm{min} / \text { week } \\
\text { for } 12 \text { weeks } \\
+ \\
\text { rehabilitation }\end{array}$ & rehabilitation & $\begin{array}{l}\text { BBS, } \\
\text { FMA-LE }\end{array}$ \\
\hline
\end{tabular}

Note: $\mathrm{EG}=$ Experimental Group; $\mathrm{CG}=$ Control Group; $\mathrm{BBS}=$ Berg Balance Scale; $\mathrm{FMA}-\mathrm{LE}=$ Fugl-Meyer Assessment Lower extremity; $\mathrm{BI}=\mathrm{Barthel}$ index; $\mathrm{PSQ}$ = F Sleep Quality Index; WHOQOL = World Health Organization Quality of Life; SF-36 = 36-item Short Form Survey; SSQOL = Stroke-Specific Quality of Life Scale; National Institutes of Health Stroke Scale. 


\begin{tabular}{|c|c|c|c|c|c|c|c|c|c|c|c|}
\hline \multirow{2}{*}{$\begin{array}{l}\text { Author } \\
\text { [Reference] }\end{array}$} & \multicolumn{2}{|c|}{ Sample } & \multicolumn{2}{|c|}{ Age range (year) } & \multicolumn{2}{|c|}{$\begin{array}{l}\text { course of disease } \\
\text { (month) }\end{array}$} & \multicolumn{2}{|c|}{$\begin{array}{l}\text { Ischemic/ } \\
\text { bleeding }\end{array}$} & \multicolumn{2}{|l|}{ Intervention } & \multirow[t]{2}{*}{ indicators } \\
\hline & EG & CG & EG & CG & EG & CG & EG & CG & EG & CG & \\
\hline $\begin{array}{l}\text { Ye et al. } \\
\text { [11] RCT }\end{array}$ & 30 & 30 & $60.17 \pm 7.21$ & $59.81 \pm 7.54$ & $1.52 \pm 0.51$ & $1.25 \pm 0.24$ & $19 / 11$ & $18 / 12$ & $\begin{array}{l}5 \times 40 \\
\text { min/week } \\
\text { for } 6 \text { weeks } \\
+ \text { Bobath } \\
\text { technologies } \\
+ \text { head hole } \\
\text { plexus } \\
\text { thorns } \\
\text { therapy }\end{array}$ & $\begin{array}{l}\text { Bobath } \\
\text { technologies } \\
\text { + head hole } \\
\text { plexus } \\
\text { thorns } \\
\text { therapy }\end{array}$ & $\begin{array}{l}\text { BBS, } \\
\text { FMA-LE, } \\
\text { BI }\end{array}$ \\
\hline $\begin{array}{l}\text { Cui et al. } \\
\text { [14] RCT }\end{array}$ & 24 & 19 & $53.67 \pm 12.98$ & $55.33 \pm 14.32$ & $1.58 \pm 0.56$ & $1.50 \pm 0.50$ & unclear & unclear & $\begin{array}{l}5 \times 30 \\
\mathrm{~min} / \text { week } \\
\text { for } 8 \text { weeks } \\
+ \\
\text { rehabilitation }\end{array}$ & rehabilitation & FMA-LE \\
\hline $\begin{array}{l}\text { Xiong et } \\
\text { al. [18] } \\
\text { RCT }\end{array}$ & 85 & 85 & $60.53 \pm 6.29$ & $59.75 \pm 6.34$ & unclear & unclear & $18 / 67$ & $18 / 67$ & $\begin{array}{l}5 \times 60 \\
\mathrm{~min} / \text { week } \\
\text { for } 12 \text { weeks } \\
+ \\
\text { rehabilitation }\end{array}$ & rehabilitation & PSQI \\
\hline $\begin{array}{l}\text { Ding et al. } \\
\text { [8] RCT }\end{array}$ & 57 & 56 & $55.37 \pm 4.71$ & $56.32 \pm 3.17$ & $6.72 \pm 2.36$ & $7.11 \pm 1.59$ & $26 / 31$ & $27 / 29$ & $\begin{array}{l}5 \times 20 \\
\mathrm{~min} / \text { week } \\
\text { for } 4 \text { weeks } \\
+ \text { balance } \\
\text { training }+ \\
\text { rehabilitation }\end{array}$ & $\begin{array}{l}\text { balance } \\
\text { training + } \\
\text { rehabilitation }\end{array}$ & $\begin{array}{l}\text { BBS, } \\
\text { FMA-LE }\end{array}$ \\
\hline $\begin{array}{l}\text { Xie et al. } \\
{[10]} \\
\text { RCT }\end{array}$ & 20 & 20 & $51.10 \pm 12.92$ & $53.95 \pm 13.00$ & $3.20 \pm 1.44$ & $3.60 \pm 1.57$ & $10 / 10$ & $10 / 10$ & $\begin{array}{l}5 \times 50 \\
\mathrm{~min} / \text { week } \\
\text { for } 3 \text { weeks } \\
\text { +Bobath } \\
\text { technologies } \\
+ \\
\text { rehabilitation }\end{array}$ & $\begin{array}{l}\text { Bobath } \\
\text { technologies } \\
+ \\
\text { rehabilitation }\end{array}$ & $\begin{array}{l}\text { BBS, } \\
\text { FMA-LE, } \\
\text { BI }\end{array}$ \\
\hline $\begin{array}{l}\text { Wei et al. } \\
\text { [17] } \\
\text { RCT }\end{array}$ & 40 & 40 & $56.1 \pm 9.2$ & $58.7 \pm 10.3$ & $15.4 \pm 3.3$ & $13.0 \pm 2.3$ & unclear & unclear & $\begin{array}{l}5 \times 30 \\
\mathrm{~min} / \text { week } \\
\text { for } 6 \text { weeks } \\
+ \\
\text { rehabilitation }\end{array}$ & rehabilitation & $\begin{array}{l}\text { BBS, } \\
\text { SSQOL, } \\
\text { PSQI }\end{array}$ \\
\hline
\end{tabular}

Note: $\mathrm{EG}=$ Experimental Group; $\mathrm{CG}=$ Control Group; BBS=Berg Balance Scale; FMA-LE = Fugl-Meyer Assessment Lower extremity; $\mathrm{BI}=\mathrm{Barthel}$ index; PSQI = F Sleep Quality Index; WHOQOL = World Health Organization Quality of Life; SF-36 = 36-item Short Form Survey; SSQOL = Stroke-Specific Quality of Life Scale; National Institutes of Health Stroke Scale.

As shown in Figure 2 and 3, the risk of total bias in the 14 selected RCTs was low. The risk of bias of random sequence generation (selection bias) was low in $55 \%$ of the studies, that of allocation concealment (selection bias) was unclear in $100 \%$ of the studies, that of blinding of participants and personnel (performance bias) was high in $100 \%$ of the studies, that of blinding of outcome assessment (detection bias) was low in $32.5 \%$ of the studies, that of incomplete outcome data (attrition bias) was low in $65 \%$ of the studies, and that of selective reporting (reporting bias) was low in $65 \%$ of the studies, and the risk of other bias was low in $100 \%$ of the studies.

\section{Meta-analysis results}

\section{The effect of Baduanjin on balance function}

Seven studies [7-13] investigated the effect of Baduanjin on balance function in stroke patients using the BBS score, including a total of 616 subjects. The higher the value of BBS, the better is the balance function. Due to the high heterogeneity of intervention measures $(P \geq 50 \%, P \leq 0.01)$ in the Baduanjin and control groups, the random-effect model was selected. The results showed that Baduanjin significantly improved the balance function compared to the control $\left[\mathrm{MD}=9.74,95 \% C /(5.32,14.15), P<0.0001, P^{2}=96 \%\right.$; Fig. 4$]$. 


\section{The effect of Baduanjin on lower limb sensorimotor function score and neural function score}

A total of 6 studies $[8-11,13,14]$ investigated the effect of Baduanjin on lower limb motor function in stroke patients using the Fugl-Meyer score. The difference in the FMA-LE lower limb function score scale was identified as the source of heterogeneity in the 6 studies, Cui et al. [14] used the FMA-LE limb function score for upper and lower limbs (lower limb data were selected for the current meta-analysis), and the rest of the studies evaluated the lower limb function. The comprehensive results showed that Baduanjin significantly improved the sensorimotor function and nerve function of lower limbs [MD $=5.37$, $95 \% \mathrm{Cl}(1.59,9.16), P=0.005, P^{2}=96 \%$; Figure 5]. Guo et al. [15] investigated the effect of Baduanjin on NIHSS score in stroke patients. The higher the NISHH score, the more severe are the symptoms. The Baduanjin group (15.97 \pm 2.84 vs. $5.25 \pm 2.54$ ) showed significantly greater improvement than the control group $(16.17 \pm 2.76$ vs. $7.66 \pm 2.49)$ in the NIHSS score. Random-effect model showed that Baduanjin significantly improved the neurological deficits [MD $=-2.41$, 95\% Cl(-3.07,-1.75), $P<0.00001$; Fig. 6].

\section{The effect of Baduanjin on the ability to perform daily activities}

Four studies $[10,11,16,17]$, including 240 subjects, investigated the influence of Baduanjin on the ability to perform daily activities in stroke patients using the Barthel scale. The comprehensive results showed that Baduanjin significantly improved the ability to perform daily activities in stroke patients $[\mathrm{MD}=14.00$, 95\% $\mathrm{Cl}(6.17,21.83), P=0.0005, P^{2}=95 \%$; Fig. 7].

\section{The effect of Baduanjin on sleep quality}

Two studies [17, 18], including 250 subjects, investigated the effects of Baduanjin on sleep quality using PSQI. The comprehensive results showed that Baduanjin significantly improved sleep quality in stroke patients [MD $=-3.08,95 \% \mathrm{Cl}(-3.90,-2.25), P<0.00001$; Fig. 8].

\section{The effect of Baduanjin on the quality of life}

Three studies [17, 19, 20], including 212 subjects, investigated the effects of Baduanjin on the quality of life in stroke patients. The comprehensive results showed that Baduanjin significantly improved the quality of life in stroke patients [MD $=31.71,95 \% \mathrm{Cl}(13.68,49.73), P=0.06$; Fig. 9]. Cai et al. [19] reported that Baduanjin exercise improved the subjective perception of the quality of life, subjective perception of health status, and quality of life in physiological, psychological, social relationship, and environmental fields in stroke patients, while no changes were observed in WHOQOL in the control group. Chen et al. [20] showed that Baduanjin combined with music rehabilitation and general rehabilitation exercises improved the quality of life of patients compared to those in the control group. Compared to the general rehabilitation group, Baduanjin combined with music improved the quality of life evaluated using SF-36. Wei et al. [17] found that after 6 months of treatment, the SSQOL score significantly improved in the Baduanjin group.

\section{The effects of different interventions on balance function in stroke patients}

Three studies $[7,8,13]$ compared the effects of Baduanjin combined with balance function training in stroke patients. Using the random-effect model, it was found that Baduanjin combined with balance function training showed a significantly greater effect on balance function than only balance function training $[\mathrm{MD}=9.07,95 \% \mathrm{Cl}(4.66,13.48), P<0.0001, P=91 \%$; Figure 10]. Three studies [10-12] compared the effects of Baduanjin combined with rehabilitation treatment and rehabilitation treatment in stroke patients, and the results showed no significant differences $[\mathrm{MD}=9.28,95 \% \mathrm{Cl}(-2.40,20.97), P=0.12, P=99 \%$; Figure 10]. One study [9] compared the effects of Baduanjin combined with routine rehabilitation training and rehabilitation training on balance and observed significant differences $[\mathrm{MD}=11.13,95 \% \mathrm{Cl}(8.55,13.71), P<0.00001, P=97 \%$; Fig. 10].

\section{The effects of different intervention durations on balance function in stroke patients}

Two studies $[8,10]$ compared the effects of Baduanjin (less than 6 weeks) on the balance function in stroke patients and found that Baduanjin (less than 6 weeks) improved the balance function [MD $=5.50,95 \% \mathrm{Cl}(3.80,7.20), P<0.00001$; Figure 11]. Five studies $[7,9,11-13]$ compared the effects of Baduanjin (more than 6 weeks) on the balance function in stroke patients, and the results showed significantly differences $[\mathrm{MD}=11.05,95 \% \mathrm{Cl}(5.01,17.10), P=0.0003$; Fig. 11].

\section{Discussion}

The results of the meta-analysis of the RCTs evaluating the rehabilitation effects of Baduanjin exercise in stroke patients showed that Baduanjin effectively improved the balance function of lower limbs, sensory-motor function, nerve function, the ability to perform daily activities, sleep quality, and quality of life.

Balance is required for walking. The current study showed that regular Baduanjin exercise improved BBS score in stroke patients. Due to the high heterogeneity of intervention measures and subgroups, we analyzed the balance intervention measures and duration separately. The results indicated that Baduanjin combined with rehabilitation treatment showed significant improvement in BBS compared to the rehabilitation training alone. Moreover, intervention duration was also an important factor affecting the balance function. Regular physical activities can not only improve the positive emotions of individuals, but also relieve anxiety, anger, depression, irritability, and other negative emotional disorders [21], relieve pressure, improve self-confidence, and improve sleep quality [22]. These may be important reasons why Baduanjin improves the quality of life in stroke patients.

Motor function is the key to improving the ability to perform daily activities in stroke patients. We found that Baduanjin combined with balance training and routine rehabilitation treatment effectively improved lower limb motor function, indicating that adjustment of breath improved the rehabilitation effect.

Baduanjin exercise improved physical activity, fitness and cardiopulmonary function. The Baduanjin exercises are symmetrical and can help improve posture. Baduanjin helps maintain body balance by transferring the body's center of gravity, moving the upper limbs, lower limbs, trunk, muscle tension, and relaxation are combined alternately. For example, "regulating the spleen and stomach requires a single lift," with both hands up and down over the head, helping to

Page 6/12 
regulate the spleen and stomach, the spleen and stomach are the important energy sources of the digestive system, which are important in promoting digestion; in "Head wagging tail to heart fire," people squatted in horse step, cerebral apoplexy patients practice improved the function of lower limbs balance and sensorimotor, because Baduanjin relaxed the muscle, and pay attention to the psychological practice skills. In "Step climbing with both hands to strengthen kidney and waist," people grabbed the toes with both hands along the leg, which strengthened kidney and waist function, improved immune system and urinary system functions.

The ability to perform daily activities guarantees basic independent functioning in stroke patients. The current study showed that regular Baduanjin exercise in stroke patients improved the ability to perform daily activities, neurological function deficit score, and self-care ability. Baduanjin can be completed within 20 minutes; it is a medium-to-low intensity aerobic exercise without any environmental constraints and helps maintain the balance using eight postures [23]. Compared with other traditional methods, Baduanjin significantly improved physical and mental health [24], physiological functions, neuromusculoskeletal functions, cognitive function, and emotional health. These may be the reasons why Baduanjin improved the ability to perform daily activities in stroke patients.

There are some limitations to the study: (1) A few studies with a high risk of bias were included, probably due to the sub-optimal judgment of the evaluators, lack of hidden allocation; (2) It was difficult to single out the effect of Baduanjin from Baduanjin combined with other behavioral interventions; (3) Most of the included studies were short-term interventions without long-term follow-up; therefore, it is unclear whether there are any long-term benefits; (4) The number of studies on sensorimotor function, nerve function, quality of life, and the ability to perform daily activities was small; (5) Only compared randomized controlled experiments were conducted, studies on the relationship between Baduanjin and rehabilitation have not been explained much.

\section{Conclusion}

Our study found that Baduanjin may be effective in the rehabilitation of stroke patients. The following suggestions are proposed based on the results and limitations of the current study: when studying the effect of Baduanjin in stroke patient rehabilitation, a high-quality of study design is necessary to understand the long-term effects of Baduanjin and help patients improve their limb rehabilitation and recover their self-care ability. Correct random allocation, clear intention analysis characteristics, hidden allocation scheme, and appropriate blindness should be employed. The intervention method, exercise duration, and exercise intensity of Baduanjin need to be standardized.

\section{Abbreviations}

EG: Experimental Group; CG: Control Group; BBS: Berg Balance Scale; FMA-LE: Fugl-Meyer Assessment Lower extremity; BI: Barthel index; PSQI: Pittsburgh Sleep Quality Index; WHOQOL: World Health Organization Quality of Life; SF-36: 36-item Short Form Survey; SSQOL: Stroke-Specific Quality of Life Scale; NIHSS: National Institutes of Health Stroke Scale.

\section{Declarations}

\section{Acknowledgements}

The authors thank Department of Physical Education, Guizhou University of Finance and Economics for data collection.

\section{Author Contributions}

$X Y X$ contributed to the conception and design of the review. $X Y X$ performed the search. $X Y X$ was involved in the screening and selection papers. $X Y X$ completed the assessment of the risk of bias. $X Y X$ analyzed and interpreted the data. $X Y X$ wrote this manuscript. $X Y X$ edited the manuscript. $X Y X$ is responsible for the overall study.

\section{Funding}

This work isn't financially supported.

\section{Availability of data and materials}

The datasets used and analysed during the current study are available from the corresponding author on reasonable request.

\section{Ethics approval and consent to participate}

This study was supported by Ethics Committee of Sports Science Experiment, Department of Physical Education, Guizhou University of Finance and Economics (NO.2021012H).

This study confirms that all methods were carried out in accordance with relevant guidelines and regulations.

\section{Consent for publication}

Not applicable.

\section{Competing interests}


The authors declare that they have no competing interest.

\section{Author details}

${ }^{1}$ Department of Physical Education, Guizhou University of Finance and Economics, Guizhou 550025, China.

\section{References}

1. Pedersen SG, Heiberg GA, Nielsen JF, et al. Validity, reliability and Norwegian adaptation of the Stroke-Specific Quality of Life (SS-QOL) scale. SAGE Open Med. 2018; 6: 1-10.

2. Roger VL, Go AS, Lloyd-Jones DM, et al. American Heart Association Statistics Committee and Stroke Statistics Subcommittee. Heart disease and stroke statistics-2012 update: a report from the American Heart Association. Circulation. 2012; 125(1): e2-e220.

3. Chinese expert consensus group on rehabilitation of acute cerebral infarction. Expert panel consensus recommendations for the management of rehabilitation after acute ischemic stroke in China. Chin J Phys Med Rehabil. 2016; 38(1): 1-6.

4. Rothwell PM, Algra A, Amarenco P. Medical treatment in acute and long-term secondary prevention after transient ischemic attack and ischemic stroke. Lancet. 2011;377(9778): 1681-1692.

5. Zheng G, Chen B, Fang Q, et al. Primary prevention for risk factors of ischemic stroke with Baduanjin exercise intervention in the community elder population: study protocol for a randomized controlled trial. Trials. 2014; 15(1): 113-122.

6. Higgins J, Green S. Cochrane handbook for systematic reviews of interventions. Chichester: WileyBlackwell, 2008; $187-235$.

7. Bai Y, Mao H, Guo J, et al. Research on Baduanjin combined with function training in improving balance function of stroke patients. Chin J Chin Med. 2011; 26(10): 1231-1232.

8. Ding Y, Guo C, Wang N, et al. Effect of Baduanjin's third part combined with conventional balance training on balance function of patients with cerebral stroke. Shangdong J Chin Med. 2019; 38(7): 673-676+704.

9. Tian H. Effect of Baduanjin on balance function in post-stroke patients. World Latest Medicine Information. 2017; 17(76): $251-252$.

10. Xie B, Yang M, Bai Y. Clinical study on the effect of Baduanjin on motor rehabilitation of stroke patients. West China Med J. 2019; 34(5): 515-519.

11. Ye T, Zhu L, Ruan Y, et al. Impacts on the lower limb motor function and balance functions in the patients of stroke at the recovery stage treated with the cluster needling technique at the head points and baduanjin exercise. World J Integr Tradit West Med. 2018; 13(3): 403-406.

12. Zhang M, Guo J, Bai Y, et al. Effect of Baduanjin on balance function among survivors of stroke. Lishizhen Med Mater Med Res. 2013; 24(10): 24382439.

13. Zhang Y, Li L. Effect of a combined intervention (Baduanjin and balance training) on balance and sensorimotor function in post-stroke patients. Shangdong J Chin Med. 2016; 35(8): 716-718.

14. Cui Y, Wang M, Yang H. Effect of health Qigong Baduanjin on motor function of stroke patients during recovery period. J Shandong Sport Univ. 2018; 34(3): 97-100.

15. Guo J, Zhang M, Bai Y, et al. Effect of Baduanjin clinical outcomes in patients with stroke. Lishizhen Med Mater Med Res. 2013; 24(12): 2941-2942.

16. Cai W, Liang C. Effects of sitting Baduanjin on the ability of activities daily living of patients with stroke sequelae in community. J Nurs Adm. 2011; 11(11): 810-811.

17. Wei Q, Zhang X. Analysis of effect of Baduanjin exercise applied in 40 cases of stroke convalescence. Beijing J Tradit Chin Med. 2019; 38(8): 816-818.

18. Xiong Z, Chen B, Fang Q, et al. Effect of Baduanjin exercise on the quality of sleep in community population at high risk of stroke. Pract Geriatr. 2018; 32(3): 259-262.

19. Cai W. Influence of sitting Baduanjin on quality of life of cerebral apoplexy patients with sequela in community. J Nurs. 2010; 24(29): 2667-2668.

20. Chen Y, Li L, Wang M, et al. Clinical study on post stroke depression treated with music therapy of five elements combine with Baduanjin. Shangdong $\mathrm{J}$ Chin Med. 2017; 36(1): 23-25.

21. Zou L, Yeung A, Quan X, et al. Mindfulness-Based Baduanjin Exercise for Depression and Anxiety in People with Physical or Mental IIInesses: A Systematic Review and Meta-Analysis. Int J Environ Res Public Health. 2018; 15(2): 321-328.

22. Chen MC, Liu HE, Huang H Y, et al. The effect of a simple traditional exercise programme (Baduanjin exercise) on sleep quality of older adults: a randomized controlled trial. Int J Nurs Stud. 2011; 49(3): 265-273.

23. Zheng G, Fang Q, Chen B, et al. Qualitative Evaluation of Baduanjin (Traditional Chinese Qigong) on Health Promotion among an Elderly Community Population at Risk for Ischemic Stroke. Evid Based Complement Alternat Med. 2015; 2015: 893215.

24. Chan JS, Li A, Ng S, et al. Adiponectin Potentially Contributes to the Antidepressive Effects of Baduanjin Qigong Exercise in Women With Chronic Fatigue Syndrome-Like Illness. Cell Transpl. 2017; 26(9): 493-501.

\section{Figures}




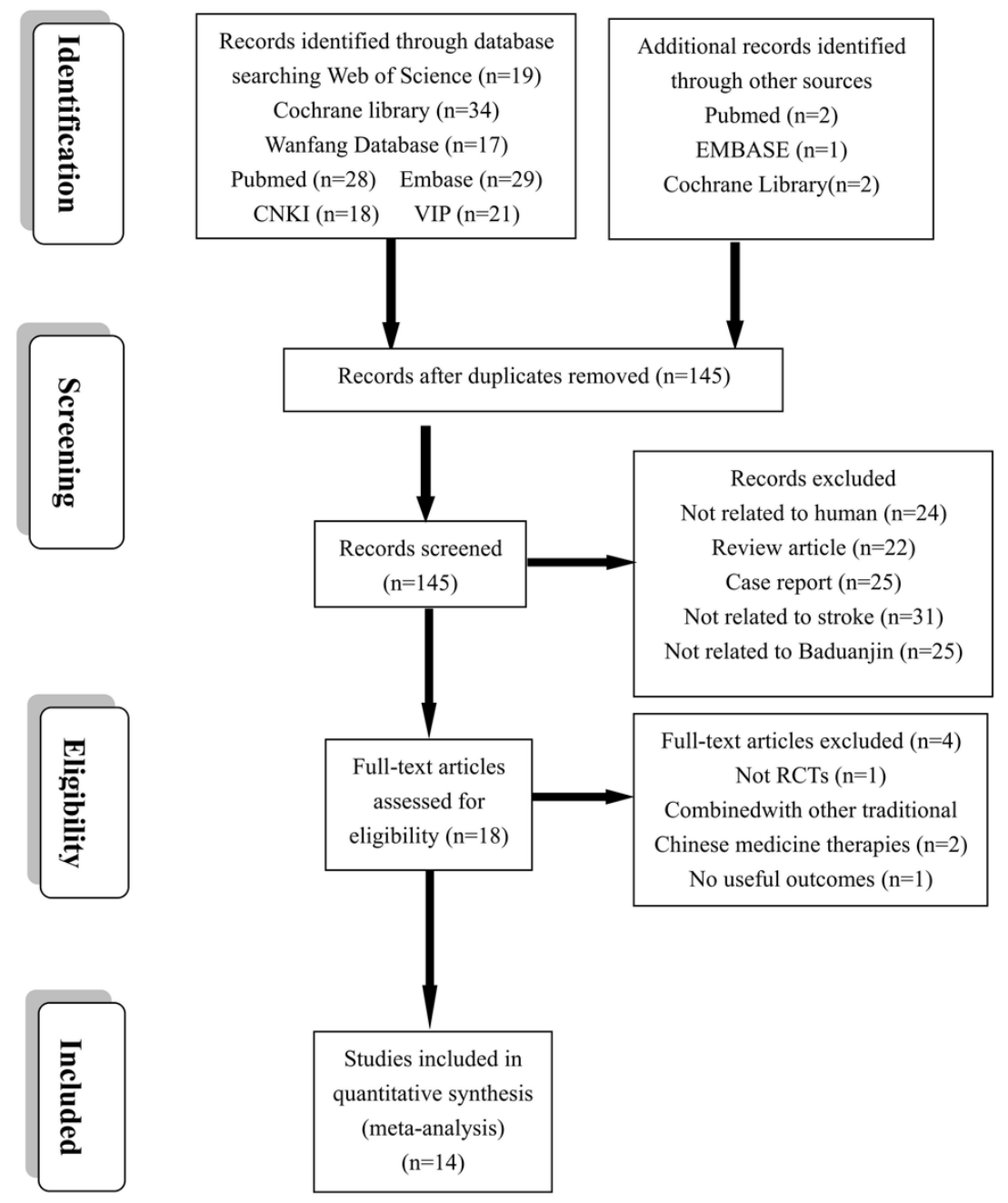

Figure 1

Flow diagram of study

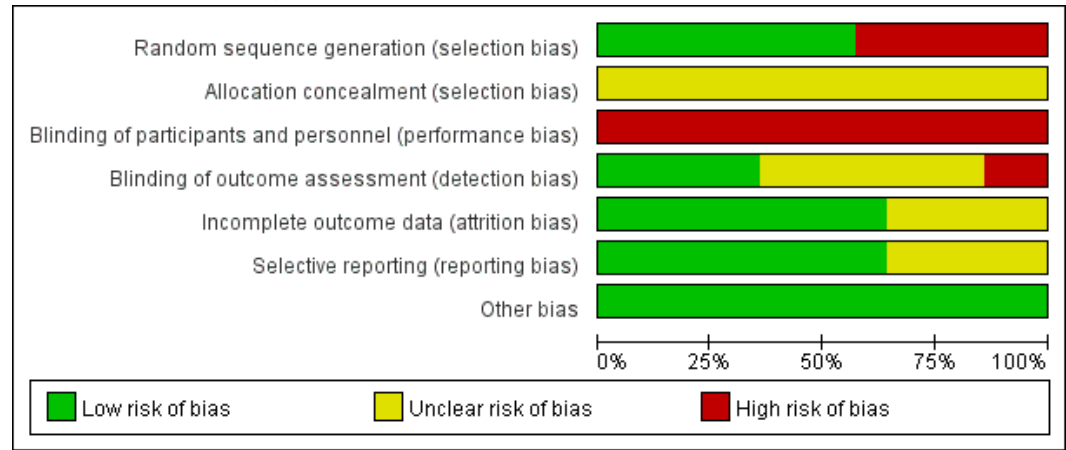

\section{Figure 2}

Overall evaluation of the risk of bias 


\begin{tabular}{|c|c|c|c|c|c|c|c|c|c|c|c|c|c|c|}
\hline 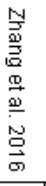 & 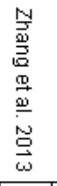 & 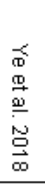 & 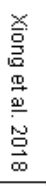 & 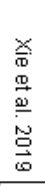 & 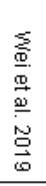 & 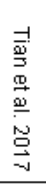 & 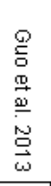 & 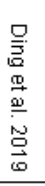 & 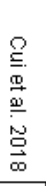 & 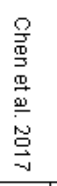 & 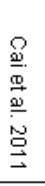 & 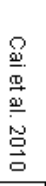 & 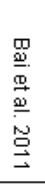 & \\
\hline$\odot$ & (1) & $\odot$ & (1) & $\odot$ & (1) & (1) & (1) & $\odot$ & (1) & $\odot$ & $\odot$ & $\odot$ & + & Random sequence generation (selection bias) \\
\hline$\sim$ & $\sim$ & $\sim$ & $\sim$ & $\bullet$ & $\sim$ & $\bullet$ & $\sim$ & $\sim$ & $\sim$ & $\sim$ & $\sim$ & $\sim$ & $\sim$ & Allocation concealment (selection bias) \\
\hline (1) & (1) & (1) & (1) & (1) & (1) & (1) & (1) & (1) & (1) & (1) & (1) & (1) & (1) & Blinding of participants and personnel (performance bias) \\
\hline$\sim$ & + & $\sim$ & $\sim$ & $\odot$ & + & $\odot$ & + & $\odot$ & $\odot$ & $\sim$ & (1) & (1) & $\sim$ & Blinding of outcome assessment (detection bias) \\
\hline$\odot$ & 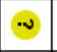 & $\odot$ & $\odot$ & $\odot$ & $\odot$ & $\odot$ & $\sim$ & $\odot$ & $\sim$ & $\odot$ & $\odot$ & $\odot$ & $\odot$ & Incomplete outcome data (attrition bias) \\
\hline$\odot$ & $\sim$ & $\odot$ & 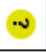 & + & 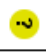 & $\odot$ & $\omega$ & $\odot$ & $\sim$ & $\odot$ & $\odot$ & $\odot$ & $\odot$ & Selective reporting (reporting bias) \\
\hline+ & + & $\odot$ & $\odot$ & + & + & $\odot$ & + & $\odot$ & $\odot$ & + & $\odot$ & $\odot$ & + & Other bias \\
\hline
\end{tabular}

Figure 3

Detailed evaluation of the risk of bias for each RCT

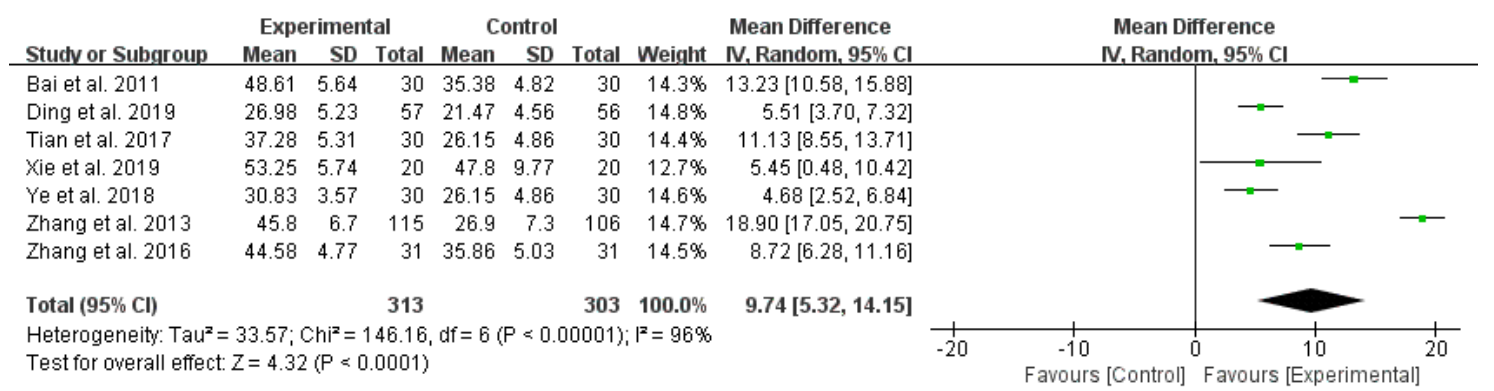

\section{Figure 4}

The effect of Baduanjin on balance function

\begin{tabular}{|c|c|c|c|c|c|c|c|c|c|c|c|c|}
\hline \multirow[b]{2}{*}{ Study or Subgroup } & \multicolumn{3}{|c|}{ Experimental } & \multicolumn{2}{|c|}{ Control } & \multirow[b]{2}{*}{ Total } & \multirow[b]{2}{*}{ Weight } & \multirow{2}{*}{$\begin{array}{l}\text { Mean Difference } \\
\text { IV, Random, } 95 \% \mathrm{Cl}\end{array}$} & \multirow{2}{*}{\multicolumn{3}{|c|}{$\begin{array}{c}\text { Mean Difference } \\
\text { IV. Random, 95\% Cl }\end{array}$}} & \\
\hline & Mean & SD & Total & Mean & SD & & & & & & & \\
\hline Cui et al. 2018 & 30.42 & 0.58 & 24 & 30.32 & 0.75 & 19 & $19.9 \%$ & $0.10[-0.31,0.51]$ & & & & \\
\hline Ding et al. 2019 & 44.68 & 4.57 & 57 & 35.96 & 5.13 & 56 & $19.1 \%$ & $8.72[6.93,10.51]$ & & & $\rightarrow-$ & \\
\hline Tian et al. 2017 & 23.31 & 7.63 & 28 & 17.13 & 5.72 & 29 & $17.1 \%$ & $6.18[2.67,9.69]$ & & & - & \\
\hline Xie et al. 2019 & 72.25 & 20.91 & 20 & 57.9 & 20.25 & 20 & $6.1 \%$ & $14.35[1.59,27.11]$ & & & & \\
\hline Ye et al. 2018 & 30.83 & 3.57 & 30 & 27.51 & 3.08 & 30 & $19.2 \%$ & $3.32[1.63,5.01]$ & & & $\rightarrow$ & \\
\hline Zhang et al. 2016 & 27.18 & 5.11 & 31 & 21.17 & 4.36 & 31 & $18.5 \%$ & $6.01[3.65,8.37]$ & & & $\rightarrow-$ & \\
\hline Total $(95 \% \mathrm{Cl})$ & & & 190 & & & 185 & $100.0 \%$ & $5.37[1.59,9.16]$ & & & & \\
\hline \multicolumn{9}{|c|}{$\begin{array}{l}\text { Heterogeneity: } \mathrm{Tau}^{2}=18.68 ; \mathrm{Chi}^{2}=126.59, \mathrm{df}=5(\mathrm{P}<0.00001) ;\left.\right|^{2}=96 \% \\
\text { Test for overall effect: } Z=2.78(\mathrm{P}=0.005)\end{array}$} & -20 & $\begin{array}{l}-10 \\
\text { urs [C }\end{array}$ & $\begin{array}{l}0 \quad 10 \\
\text { Favours [E: }\end{array}$ & $\begin{array}{c}20 \\
\text { mental] }\end{array}$ \\
\hline
\end{tabular}

Figure 5

The effect of Baduanjin on Fugl-Meyer lower limb function score (condition 1)

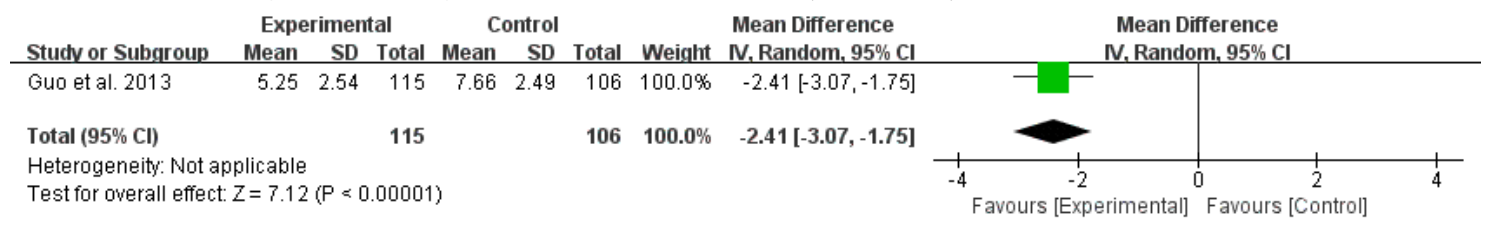

Figure 6

The effect of Baduanjin on neural deficits function score (condition 2)

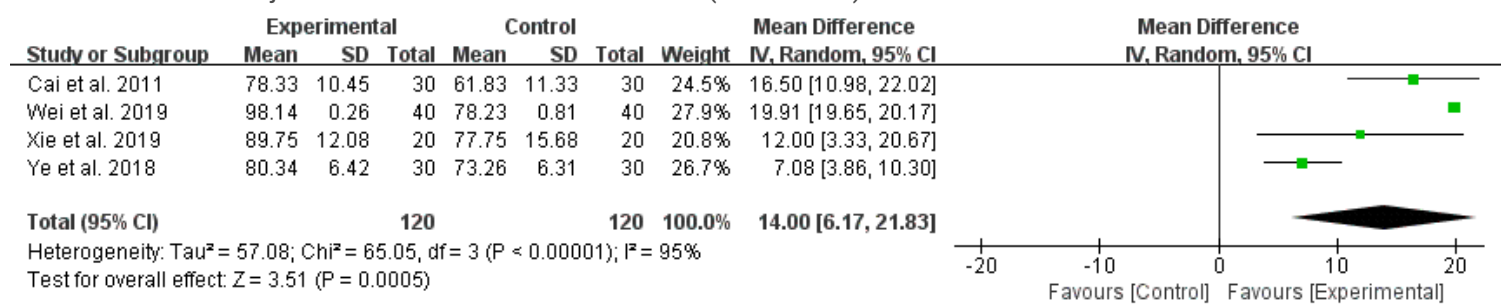


Figure 7

The effect of Baduanjin on the Barthel index

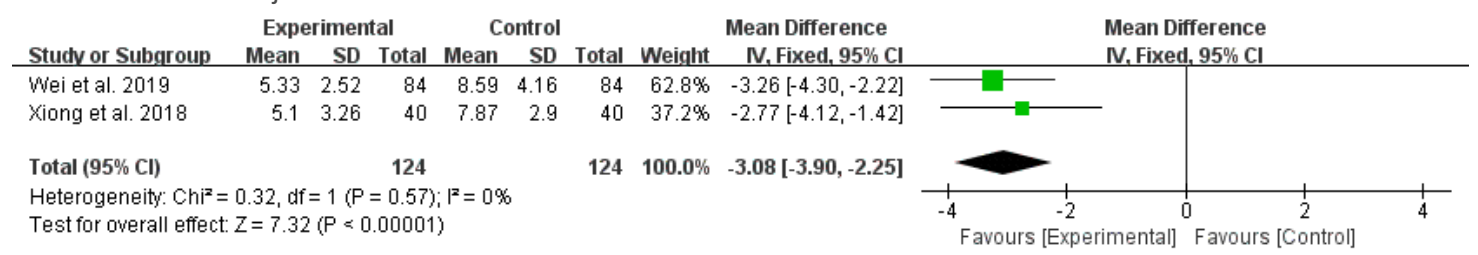

\section{Figure 8}

The effect of Baduanjin on PSQI

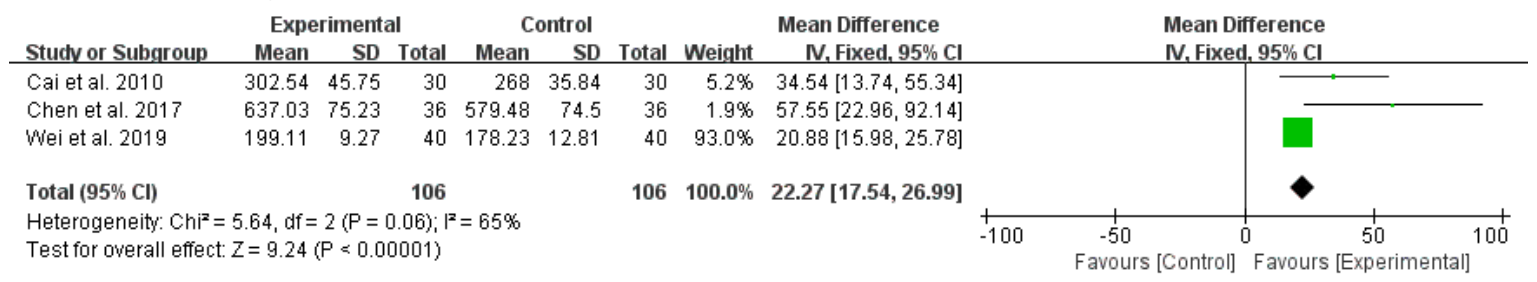

\section{Figure 9}

The effect of Baduanjin on the quality of life

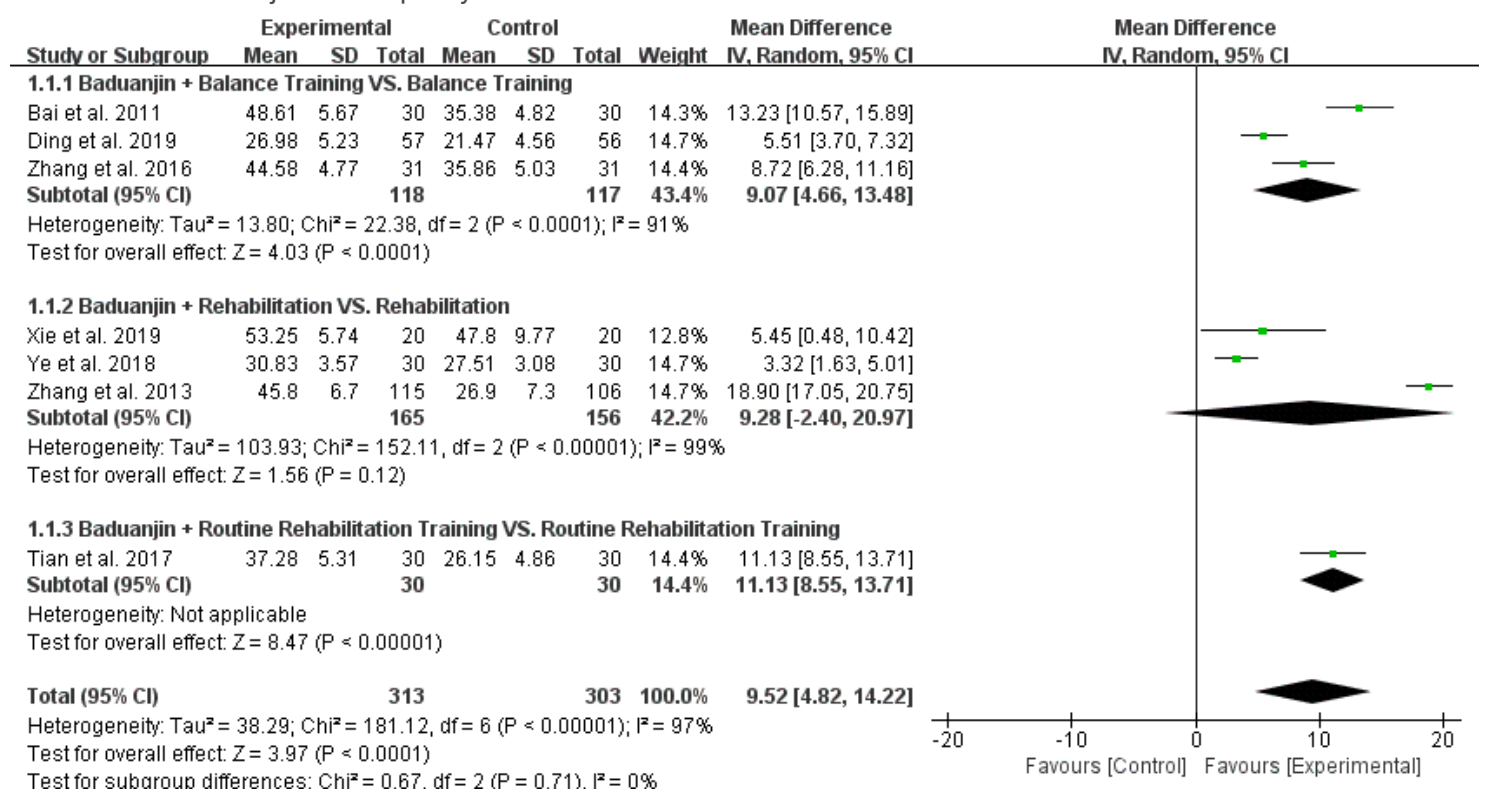

\section{Figure 10}

The effects of different interventions on balance function in stroke patients 
Experimental Control Mean Difference Mean Difference

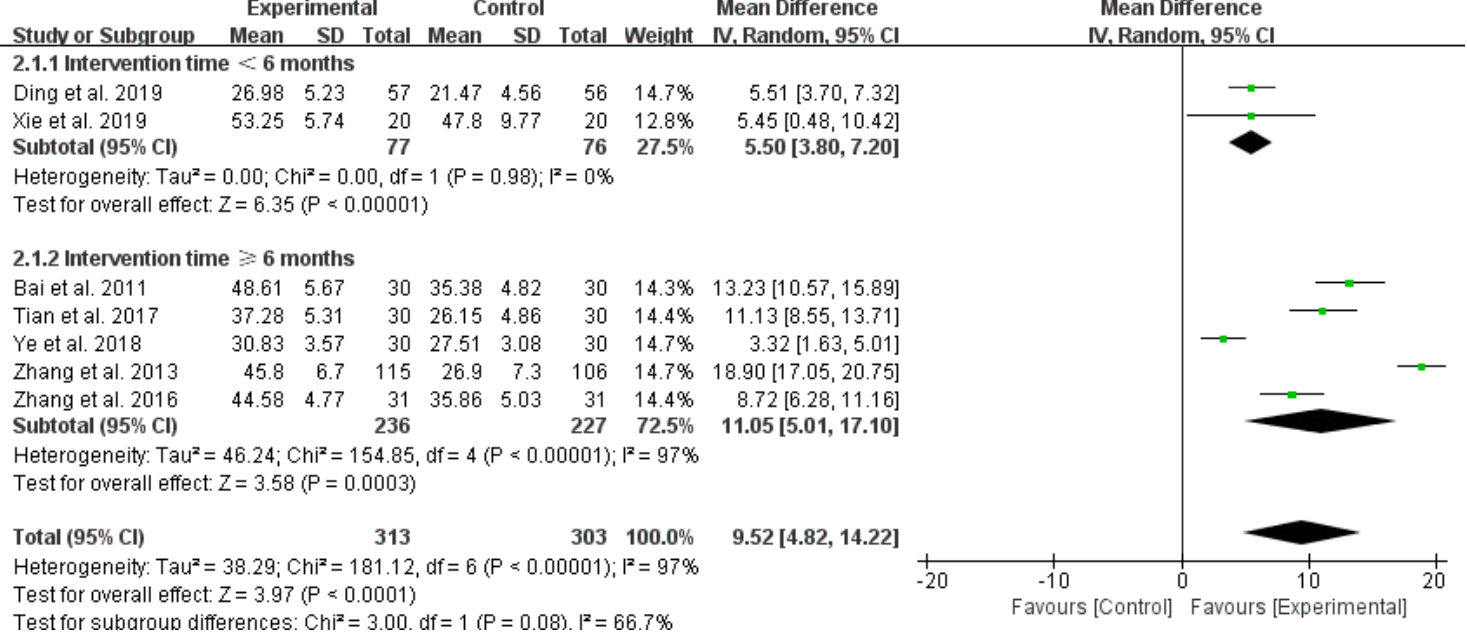

\section{Figure 11}

The effects of different intervention durations on balance function in stroke patients 